\title{
Trajectoires de moindre action et temps de vie pour trois modes de désintégration du noyau ${ }^{234} \mathrm{U}$
}

\author{
T. Benfoughal $\left({ }^{1, *}\right)$ et M. Mirea $\left({ }^{2}\right)$ \\ $\left({ }^{1}\right)$ Institut de Physique Nucléaire, 91406 Orsay Cedex, France \\ $\left({ }^{2}\right)$ Institut de Physique Atomique, P.O. Box MG-6, Bucarest, Roumanie
}

(Reçu le 7 mai 1996, accepté le 11 juillet 1996)

PACS.23.70.+j - Heavy-particle decay

Résumé. - Les trajectoires optimales de fission pour trois modes de désintégration du noyau ${ }^{234} \mathrm{U}$ sont obtenues à l'aide d'une méthode numérique. L'intégrale WKB est minimisée en fonction des coordonnées généralisées qui caractérisent le col et l'élongation. Un terme d'énergie qui prend en considération les effets dus à une surface diffuse du noyau est ajouté à l'énergie de déformation calculée dans le cadre du modèle macroscopique des systèmes binaires avec différentes densités de charge. Les masses effectives sont calculées avec des coordonnées généralisées choisies pour inclure les variations de l'asymétrie durant le processus nucléaire. Des calculs ont été effectués pour obtenir les trajectoires de fission dans un grand domaine d'asymétrie de masse rendant compte de la fission froide, de l'émission spontanée de noyaux lourds et de la désintégration alpha. Des estimations de temps de vie sont reproduites.

\begin{abstract}
A numerical method is used to obtain the optimum cold fission paths for three decay modes of ${ }^{234} \mathrm{U}$. The WKB-integral is minimized with respect the necking and the elongation. A term which takes into account the effect of the diffuse surface is added to the deformation energy in the framework of the macroscopic model of binary system with different charge densities. The effective masses are computed with more correct coordinates of deformation in order to include the variations of the asymmetry during the processes. Calculations are performed to obtain the fission trajectories in a large range of mass-asymmetry including cold fission, cluster emission and alpha-decay. Estimations of $T_{1 / 2}$ are reported.
\end{abstract}

\section{Introduction}

Les modèles basés sur l'extrapolation de la théorie de la fission nucléaire dans le domaine des grandes asymétries de masse ont réussi à prédire le processus de radioactivité exotique $[1,2]$ Trois modèles phénoménologiques, basés sur une description unitaire de la désintégration alpha, l'émission des ions lourds et la fission froide, ont été publiés : la théorie de la fragmentation, le modèle de fission superasymétrique analytique et le modèle de fission superasymétrique numérique. Tous ces modèles présentent un degré de liberté fixé, c'est-à-dire l'asymétrie de masse pendant le processus de fission et, en plus, considèrent que la forme du système nucléaire est donnée par une paramétrisation très simple, celle de deux sphères en intersection (recouvrement partiel). Le seul degré de liberté qui peut évoluer est l'élongation. Dans le cas du modèle

(*) Auteur auquel doit être adressée la correspondance (e-mail : benfoughal@ipncls.in2p3.fr)

(C) Les Éditions de Physique 1996 
superasymétrique numérique, une variation des densités de charge des fragments est prise en considération, mais elle présente une évolution prescrite a priori par rapport à l'élongation. Malgré ces approximations très sévères, des calculs de temps de vie, obtenus avec le modèle superasymétrique analytique et effectués en prenant en compte une formule semi-empirique pour la dépendance de l'énergie macroscopique de vibration du point zéro en fonction des masses des fragments sont en accord, à un ordre de grandeur près, avec l'expérience. Ces résultats paraissent étonnants, compte tenu du fait que les estimations de temps de vie pour le processus de fission présentent encore des désaccords de plusieurs ordres de grandeurs (jusqu'à 5 ordres). Les deux modèles de fission superasymétrique considèrent que les valeurs des temps de vie sont données par la probabilité de franchir la barrière de potentiel unidimensionnelle pendant la désintégration. La seule différence est que le modèle analytique n'utilise pas une expression réaliste de l'énergie de déformation pour les états de pré-scission et l'approxime avec un polynôme, tandis que la masse effective est considérée comme constante. Ces simplifications permettent une forme analytique pour l'intégrale d'action, donc un temps de calcul réduit et des estimations rapides des temps de vie. Pour obtenir de meilleures prédictions avec moins de dépendances semi-empiriques, des calculs qui prennent en compte les variations des principaux degrés de liberté rencontrés dans les processus de désintégration nucléaire ont été abordés [3-5]. Les résultats ont montré que l'introduction, dans ce modèle, de la dépendance par rapport à la taille du col peut conduire à des estimations de temps de vie très réalistes, tandis que la dépendance par rapport à la variation du paramètre caractérisant l'asymétrie de masse est beaucoup moins importante pour une voie de désintégration donnée $[5,6]$. Dans cet article, on se propose d'estimer les temps de vie pour trois modes de désintégration du noyau ${ }^{234} \mathrm{U}$ en calculant les trajectoires de fission dans l'espace multidimensionnel des configurations. Pour cela, on procède à la minimisation de l'intégrale WKB (Wentzel - Kramers - Brillouin) par rapport aux coordonnées caractérisant le col et l'élongation. Une dépendance selon l'asymétrie de masse est prise en compte, car on impose à la paramétrisation de la forme nucléaire la condition que le volume du fragment émis pendant le processus de fission ne dépasse jamais son volume final pour une voie de fission donnée. On utilise une généralisation du formalisme mathématique [7] qui permet d'approximer la trajectoire de fission au moyen d'un nombre donné de valeurs des coordonnées généralisées et une fonction de lissage par splines. On a introduit dans le modèle des systèmes binaires avec densités de charge différentes un terme de correction qui tient compte de la diffusivité de la surface nucléaire.

\section{Paramétrisation de la forme nucléaire}

Pendant le processus de désintégration, la surface nucléaire est approximée par deux sphères reliées, sans discontinuités de premier ordre, par une surface obtenue par la rotation, autour de l'axe de symétrie, d'un arc de cercle [6], comme représenté sur la figure 1. La distance entre le centre de l'arc de cercle de la surface médiane et l'axe de symétrie est notée $\rho_{3}$. L'expression de cette dernière est choisie de telle sorte qu'elle prenne une valeur positive si les formes obtenues présentent un col étroit ou, au contraire, une valeur négative si les formes nucléaires sont gonflées (c'est-à-dire présentent une forme proche de celle du diamant) dans la région médiane.

Le processus se produisant à très basse énergie, nous cherchons surtout à mettre en évidence le rôle joué par les trois coordonnées généralisées les plus pertinentes : élongation, striction (ou taille du col) et asymétrie de masse. C'est la raison pour laquelle les coordonnées généralisées qui caractérisent les déformations de chaque fragment ne sont pas prises en considération. Pour simplifier les calculs, l'asymétrie de masse est donnée par le rayon du fragment léger, $R_{2}$, au lieu du conventionnel paramètre équivalent $\eta=\frac{A_{1}-A_{2}}{A_{0}}$, où $A_{0}, A_{1}$ et $A_{2}$ sont, respectivement, 

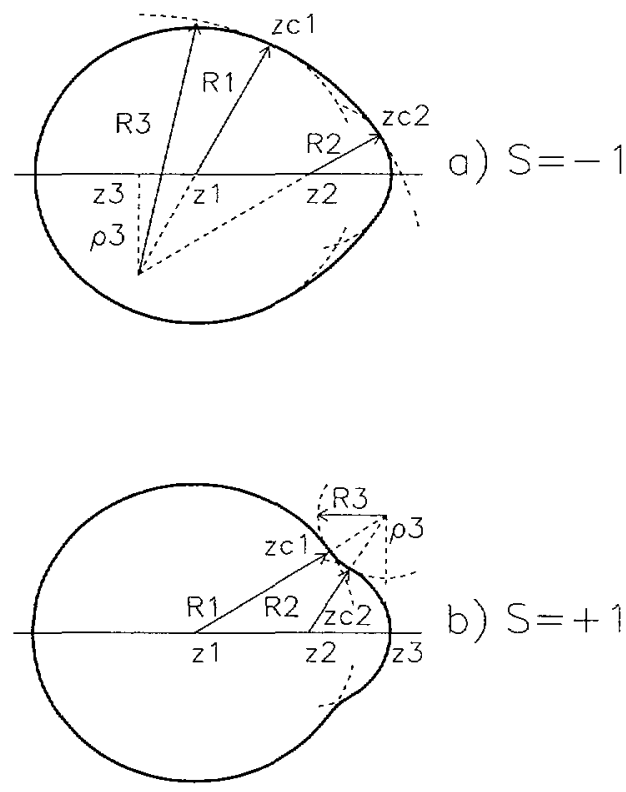

Fig. 1. - Paramétrisation de la forme nucléaire

[Nuclear shape parametrization.]

le nombre de masse du noyau parent, du noyau descendant et du fragment émis. (Nous conserverons dorénavant ces indices de notation dans tout cet article). Le col est caractérisé par le rayon $R_{3}$ de l'arc de cercle, ou bien, alternativement, pour les cas où $R_{3}$ prend des valeurs élevées (ou quand les formes nucléaires sont gonflées) par le paramètre $C=S / R_{3}$, où $S=$ sign $\left(\rho_{3}\right)$ est une notation pour le signe : les formes en diamant sont obtenues pour $S=-1$ et l'existence d'un col pour $S=+1$. En imposant des valeurs aux trois degrés de liberté pertinents retenus et en respectant la condition de conservation de volume, on obtient des valeurs pour tous les paramètres qui contribuent à définir la forme nucléaire : $R_{1}$ qui représente le rayon du noyau descendant, $z_{1}, z_{2}, z_{3}$ qui représentent les positions, sur l'axe de symétrie $\mathrm{O} z$, des centres des sphères de rayons $R_{1}$ et $R_{2}$ et de l'arc de cercle de rayon $R_{3}$, et, enfin, $z_{\mathrm{c}_{1}}$ et $z_{\mathrm{c}_{2}}$ qui représentent les positions, sur l'axe $\mathrm{O} z$, des surfaces de séparation entre la surface médiane et les deux fragments. Du fait de la symétrie axiale, l'équation de la surface en coordonnées cylindriques est la suivante :

$$
\begin{gathered}
\rho_{\mathrm{s}}=\sqrt{R_{1}^{2}-\left(z-z_{1}\right)^{2}}, \quad z \leq z_{\mathrm{c}_{1}} \\
\rho_{\mathrm{s}}=\rho_{3}-S \sqrt{R_{3}^{2}-\left(z-z_{3}\right)^{2}}, \quad z_{\mathrm{c}_{1}} \leq z \leq z_{\mathrm{c}_{2}} \\
\rho_{\mathrm{s}}=\sqrt{R_{2}^{2}-\left(z-z_{2}\right)^{2}}, \quad z>z_{\mathrm{c}_{2}}
\end{gathered}
$$

Quand $S=\operatorname{sign}\left(\rho_{3}\right)$ prend la valeur -1 , le volume $V_{2}$ du fragment émis est toujours calculé en intégrant sur l'intervalle $\left[z_{\mathrm{c}_{2}}, z_{2}+R_{2}\right]$. Pour $S=+1, V_{2}$ est calculé dans l'intervalle $\left[z_{\mathrm{c}_{2}}, z_{2}+R_{2}\right]$, dans le cas où $z_{\mathrm{c}_{2}} \leq z_{3}$ et dans l'intervalle $\left[z_{3}, z_{2}+R_{2}\right]$, dans le cas où $z_{\mathrm{c}_{2}}>z_{3}$. Lorsque le volume du fragment émis, calculé dans le domaine spécifié plus haut, est plus grand que son volume 
final $V_{2 \mathrm{f}}=\frac{4 \pi}{3} r_{0} A_{2}^{\frac{1}{3}}$, une seconde équation est résolue pour avoir l'égalité $V_{2}\left(R, R_{3}\right)=V_{2 \mathrm{f}}$, qui donne une nouvelle valeur pour $R_{2}$. Cette valeur ne coïncide pas avec $R_{2 \mathrm{f}}$ (le rayon final du fragment émis, égal à $\left.r_{0} A_{2}^{\frac{1}{3}}\right)$. Cette situation se retrouve au voisinage du point de scission. Pour cette raison, on doit également calculer les masses effectives par rapport à $R_{2}$. Pour cela, on a besoin des valeurs des dérivées partielles $\frac{\partial R_{2}}{\partial R}$ qui dépendent de $R$ et $R_{3}$ (ou $C$ ). Le calcul de ces valeurs est effectué numériquement.

Le volume du noyau descendant est alors $V_{1}=V_{0}-V_{2}$. Pendant la déformation, le volume total est conservé, $V_{0}=\frac{4 \pi}{3} r_{0} A_{0}^{\frac{1}{3}}$. La constante du rayon est, dans ce travail, $r_{0}=1,16 \mathrm{fm}$. Le cas particulier de deux sphères en intersection est obtenu pour $R_{3}=0$.

Dans les calculs des trajectoires, l'élongation normalisée $R_{n}=\left(R-R_{2}\right) /\left(R_{\mathrm{f}}-R_{2}\right)$ - où $R_{\mathrm{f}}=R_{1 \mathrm{f}}+R_{2 \mathrm{f}}$ représente la somme des rayons finaux des deux fragments formés après la désintégration; c'est donc l'élongation au point de scission pour deux sphères en contact, et $R_{2}=R_{0}-R_{2 \mathrm{f}}$ représente le moment où le fragment émis commence à sortir du noyau parent - est préférée, car les calculs s'en trouvent simplifiés. Le moment initial du processus de fission est caractérisé par la valeur $R_{n}=0$ et le point de scission pour la paramétrisation de la forme donnée par deux sphères tangentes correspond à $R_{n}=1$.

\section{Le tenseur d'inertie nucléaire}

Pour calculer l'intégrale d'action, on a besoin de deux quantités : le tenseur d'inertie nucléaire et l'énergie de déformation. Une méthode très connue de calcul du tenseur des masses effectives est l'approximation Werner-Wheeler. Dans cette approximation, on suppose que ce tenseur est une mesure du mouvement collectif et on cherche à le déterminer dans le cadre d'un modèle hydrodynamique pour un fluide incompressible, sans viscosité et irrotationel. En effectuant les calculs en coordonnées cylindriques, cette approximation équivaut à dire que la dérivée, par rapport au temps, de $z$, pour déplacer un élément de masse nucléaire, est indépendante de $\rho$ et que la dérivée de $\rho$, par rapport au temps, dépend linéairement de $\rho$ :

$$
\begin{array}{r}
\dot{z}=\sum_{\imath=1}^{n} A_{2}(z ; \beta) \dot{\beta}_{\imath} \\
\dot{\rho}=\frac{\rho}{\rho_{\mathrm{s}}} \sum_{\imath=1}^{n} B_{\imath}(z ; \beta) \beta_{\imath}
\end{array}
$$

où $\rho_{\mathrm{s}}$ est la valeur de la coordonnée $\rho$ sur la surface nucléaire.

Finalement, on obtient l'expression suivante pour les composantes du tenseur d'inertie :

$$
B_{\imath \jmath}=\pi \sigma_{m} \int_{z_{\min }}^{z_{\max }} \rho_{\mathrm{s}}^{2}\left(A_{2} A_{\jmath}+\frac{1}{2} B_{\imath} B_{\jmath}\right) \mathrm{d} z+B_{\imath \jmath}^{\mathrm{corr}}
$$

$\sigma_{m}=3 m /\left(4 \pi r_{0}^{3}\right)$ étant la densité nucléaire où $m$ est la masse du nucléon. Les fonctions $A$ et $B$ résultent des hypothèses de l'approximation Werner-Wheeler et des conditions aux frontières qui imposent l'annulation de la composante normale de la vitesse sur la surface nucléaire. Ces fonctions ont les expressions suivantes :

$$
\begin{aligned}
& A_{\imath \mathrm{l}}(z ; \beta)=-\frac{1}{\rho_{\mathrm{s}}^{2}} \frac{\partial}{\partial \beta_{\imath}} \int_{z_{\min }}^{z} \rho_{\mathrm{s}}^{2} \mathrm{~d} x \\
& A_{\imath \mathrm{r}}(z ; \beta)=-\frac{1}{\rho_{\mathrm{s}}^{2}} \frac{\partial}{\partial \beta_{\imath}} \int_{z}^{z_{\max }} \rho_{\mathrm{s}}^{2} \mathrm{~d} x
\end{aligned}
$$




$$
B_{\imath}(z ; \beta)=-\frac{1}{2} \rho_{\mathbf{s}} \frac{\partial A_{2}}{\partial z}
$$

où $z_{\min }, z_{\max }$ sont les valeurs des positions des extrémités du noyau sur l'axe de symétrie. Les indices $l$ et $r$ signifient que les fonctions sont calculées, respectivement, pour la partie gauche et pour la partie droite du noyau. Enfin, le dernier terme :

$$
B_{2 \jmath}^{\text {corr }}=-\left(\pi^{2} \sigma / V\right) \int_{z_{\min }}^{z_{\max }} \rho_{\mathrm{s}}^{2} A_{2} \mathrm{~d} z \int_{z_{\min }}^{z_{\max }} \rho_{\mathrm{s}}^{2} A_{\jmath} \mathrm{d} z
$$

représente une correction due au mouvement du centre de masse qui assure l'obtention d'une masse réduite correcte pour une configuration du noyau réalisée par deux sphères disjointes ou ayant un point de tangence. Ce terme est non nul si l'origine de l'axe de symétrie $\mathrm{O} z$ ne coïncide pas avec le centre de masse du système nucléaire. Dans un espace multidimensionnel des configurations du système nucléaire, quand la forme du noyau est décrite par un ensemble de $n$ paramètres indépendants, il est possible d'obtenir l'inertie le long de la trajectoire dynamique au moyen de la relation suivante :

$$
B(q)=\sum_{i, \jmath=1}^{n} B_{2 \jmath}(\beta) \frac{\partial \beta_{2}}{\partial q} \frac{\partial \beta_{3}}{\partial q}
$$

Ici, $q$ représente une variable indépendante (par exemple, l'élongation $R$ ) par rapport à laquelle on veut déterminer le comportement des coordonnées généralisées : $\beta_{2}=\beta_{2}(q)$. Dans notre cas, on doit étudier les variations des paramètres $B$ par rapport aux trois paramètres indépendants $R, C$ et $R_{2}$ qui caractérisent nos degrés de liberté. Pour la désintégration alpha du noyau ${ }^{234} \mathrm{U}$, on a représenté, sur les figures 2 à 4 , les variations des coefficients des masses effectives diagonales. Pour l'émission du ${ }^{28} \mathrm{Mg}$, les mêmes composantes sont représentées sur les figures 5 à 7 , tandis que pour la fission froide - avec, pour fragment léger, ${ }^{100} \mathrm{Zr}-$, on a les comportements correspondants sur les figures 8 à 10 .

Une étude critique des principaux comportements des éléments de ce tenseur peut être réalisée si on prend en compte le fait que la minimisation de l'intégrale d'action exige des trajectoires caractérisées également par des inerties aussi faibles que possible.

Les composantes non-diagonales ne présentent pas une grande importance ; car leurs valeurs sont très faibles, ce qui conduit même certains auteurs [3] à les négliger dans leurs calculs. Comparativement à d'autres résultats publiés [8], obtenus avec $R_{2 \mathrm{f}}$ comme paramètre indépendant utilisé pour décrire l'asymétrie de masse, les comportements présentés ici sont un peu différents.

On ne retrouve pas la décroissance très "prégnante" dans la variation de $B_{R R}$ (Figs. 2, 5 et 8 ) par rapport à l'élongation quand le volume $V_{2}$ égale $V_{2 \mathrm{f}}$. Les valeurs de $B_{R R}$ sont nulles quand l'élongation normalisée est égale à zéro et deviennent égales à la masse réduite $\mu=\frac{A_{1} A_{2}}{A_{0}}$ après la scission, pour la configuration de deux sphères disjointes. Pour la désintégration alpha, on observe que $B_{R R}$ prend des valeurs plus grandes quand $C$ diminue (c'est-à-dire, pour des valeurs élevées du rayon du col, équivalent à des formes en diamant). Donc, la désintégration alpha va être plutôt caractérisée pour une paramétrisation donnée par deux sphères en intersection tandis que les autres modes de désintégration vont être favorisés par une paramétrisation de la surface nucléaire rendant compte des formes en diamant. Les masses effectives par rapport à $C$ (Figs. 3, 6 et 9) sont nulles quand $R_{3}=0 \mathrm{fm}$ (ou quand $C$ tend vers l'infini) et quand on retrouve la configuration de deux sphères disjointes, réalisée après la scission. Les valeurs de ce paramètre diminuent pour les formes caractérisées par de faibles valeurs de $C$ lorsque la masse du fragment émis augmente. Ce paramètre favorise donc les formes en diamant pour l'émission spontanée d'ions lourds et la fission froide par rapport à la désintégration alpha. 


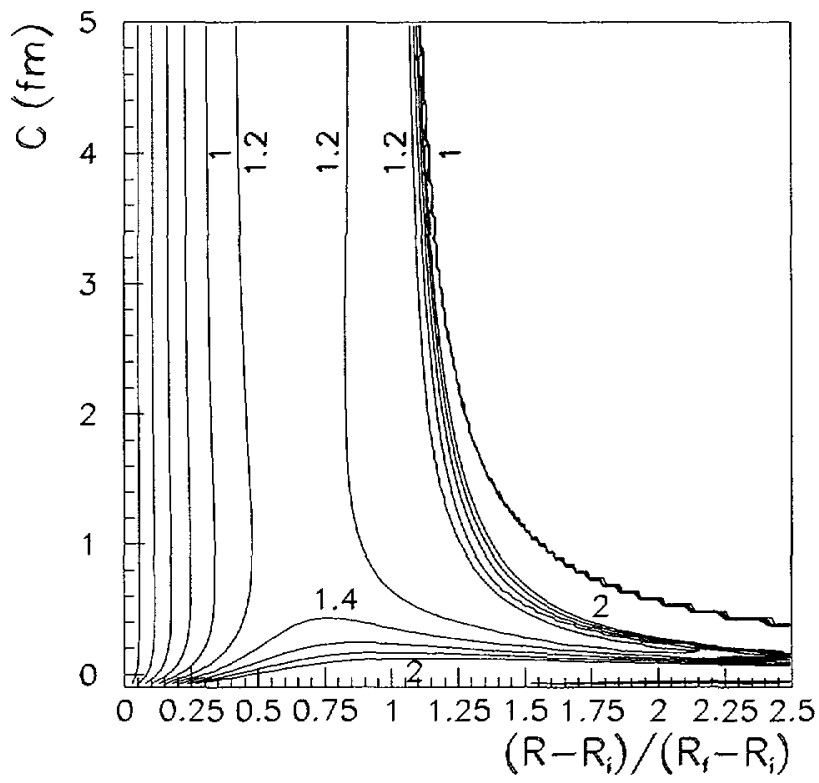

Fig. 2. - Courbes de niveaux pour la composante inertielle $B_{R R} / \mu$ dans le cas de la désintégration alpha du noyau parent ${ }^{234} \mathrm{U}$ par rapport aux deux coordonnées généralisées $C$ et $\left(R-R_{2}\right) /\left(R_{\mathrm{f}}-R_{2}\right)$. Les valeurs de cette composante sont nulles pour le noyau parent considéré sphérique et sont égales à la masse réduite $\mu$ quand les deux fragments obtenus après la désintégration sont bien séparés. Le pas entre deux valeurs consécutives des niveaux est de 0,2 unités. Quelques valeurs sont représentées auprès de certains niveaux pour guider l'œil.

[Contour plots of the inertia component $B_{R R} / \mu$ for the alpha-decay of the parent nucleus ${ }^{234} U$ with respect two generalized coordinates $C$ and $\left(R-R_{\imath}\right) /\left(R_{\mathrm{f}}-R_{2}\right)$. The values of this component are zero for the spherical parent nucleus and equal the reduced mass $\mu$ when the two fragments are separated after the decay. The step between two levels is 0.2 units. Some values are plotted in the vicinity of some levels to guide the eyes.]

En ce qui concerne le comportement des masses $B_{R_{2} R_{2}}$ (Figs. 4, 7 et 10), on observe une augmentation de ce paramètre avec l'élongation pour arriver finalement, avec un accroissement de $R$, à des valeurs proches de l'infini. Ceci s'explique car on obtient, après la scission, une valeur finale $R_{2 \mathrm{f}}$ du fragment émis, et toute variation de ce paramètre devrait se produire, dorénavant, grâce à la compressibilité de la matière nucléaire ; car il n'y a plus aucun contact entre les fragments pour effectuer un transfert de masse. Mais notre modèle est valable pour un fluide incompressible, et dans ces conditions, on ne peut plus avoir de modifications. Par conséquent, la masse effective par rapport à $R_{2}$ devient infinie. On observe aussi que $B_{R_{2} R_{2}}$ augmente quand $C$ diminue, mais ce comportement devient moins sensible avec l'augmentation de la masse du fragment émis. Par conséquent, ce paramètre favorise plutôt les formes en diamant pour l'émission des fragments lourds par rapport à la désintégration alpha.

Les effets de viscosité pour un tel système fissionnable sont négligeables ; ce fait est justifié par la valeur expérimentale de l'énergie cinétique très proche de la chaleur de désintégration $Q$. Par conséquent, pour ce type de processus, sans dissipation, on ne prend pas en considération le tenseur des forces de friction. 


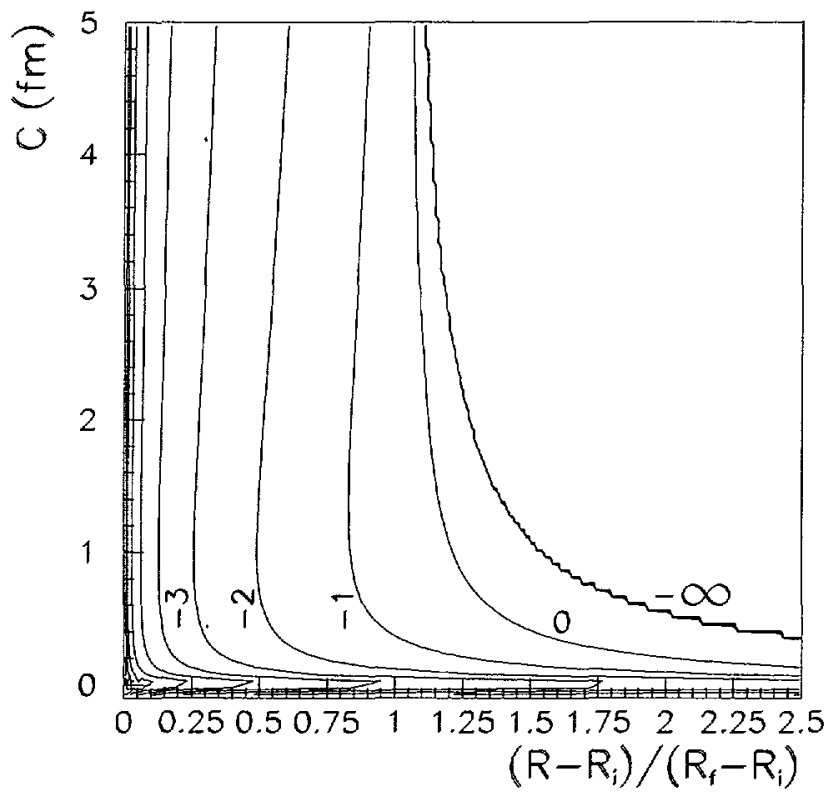

Fig. 3. - Courbes de niveaux pour la composante inertielle $\log \left(B_{C C} / \mu\right)$ dans le cas de la désintégration alpha du noyau parent ${ }^{234} \mathrm{U}$ par rapport aux deux coordonnées généralisées $C$ et $\left(R-R_{\imath}\right) /\left(R_{\mathrm{f}}-R_{\imath}\right)$. Les valeurs de cette composante sont nulles (c'est-à-dire, $-\infty$ en échelle logarithmique) pour le noyau parent considéré sphérique et quand les deux fragments obtenus après la désintégration sont bien séparés. Le pas entre deux valeurs consécutives des niveaux est de 1 unité. Quelques valeurs sont représentées près de certains niveaux pour guider l'œil.

[Contour plots of the inertia component $\log \left(B_{C C} / \mu\right)$ for the alpha-decay of the parent nucleus ${ }^{234} \mathrm{U}$ with respect two generalized coordinates $C$ and $\left(R-R_{2}\right) /\left(R_{\mathrm{f}}-R_{2}\right)$. The values of this component are zero (i.e.,$-\infty$ in a logarithmic scale) for the spherical parent nucleus and when the two fragments are separated after the decay. The step between two levels is 1 unit. Some values are platted in the vicinity of some levels to guide the eyes.]

\section{4. Énergie de déformation}

L'énergie de déformation est calculée dans le cadre du modèle de la goutte liquide pour une interaction du type Yukawa-plus-exponentielle [9], en utilisant le formalisme des systèmes binaires avec des densités de charge différentes [10]. L'asymétrie de charge est considérée constante jusqu'au moment où l'élongation normalisée $R_{n}=\left(R-R_{2}\right) /\left(R_{\mathrm{f}}-R_{2}\right)$ atteint la valeur $R_{n} \approx 0,7$, puis varie linéairement pour chacun des deux fragments naissants jusqu'à leurs valeurs finales quand $R_{n}=1$. Dans ce modèle, les énergies de déformation, dues aux potentiels nucléaire $E_{\mathrm{n}}$ et électrostatique $E_{\mathrm{c}}$, sont obtenues avec la somme de trois termes, c'est-à-dire, les deux énergies propres des fragments et l'énergie d'interaction :

$$
\begin{aligned}
& E_{\mathrm{n}} / E_{\mathrm{n}}^{0}=\left(c_{\mathrm{s}_{1}} / c_{\mathrm{s}}\right) B_{\mathrm{n}_{1}}+\left[\left(c_{\mathrm{s}_{1}} c_{\mathrm{s}_{2}}\right)^{1 / 2} / c_{\mathrm{s}}\right] B_{\mathrm{n}_{12}}+\left(c_{\mathrm{s}_{2}} / c_{\mathrm{s}}\right) B_{\mathrm{n}_{2}} \\
& E_{\mathrm{c}} / E_{\mathrm{c}}^{0}=\left(\rho_{1 \mathrm{e}} / \rho_{0 \mathrm{e}}\right)^{2} B_{\mathrm{c}_{1}}+\left(\rho_{1 \mathrm{e}} \rho_{2 \mathrm{e}} / \rho_{0 \mathrm{e}}^{2}\right) B_{\mathrm{c}_{12}}+\left(\rho_{2 \mathrm{e}} / \rho_{0 \mathrm{e}}\right)^{2} B_{\mathrm{c}_{2}}
\end{aligned}
$$

où $E_{\mathrm{n}}^{0}$ et $E_{\mathrm{c}}^{0}$ correspondent aux formes sphériques, $\rho_{2 e}$ sont les densités de charge et

$$
c_{\mathrm{s} \imath}=a_{\mathrm{s}}\left[1-\kappa_{\mathrm{s}}\left(N_{\imath}-Z_{\imath}\right)^{2} / A_{\imath}^{2}\right]
$$




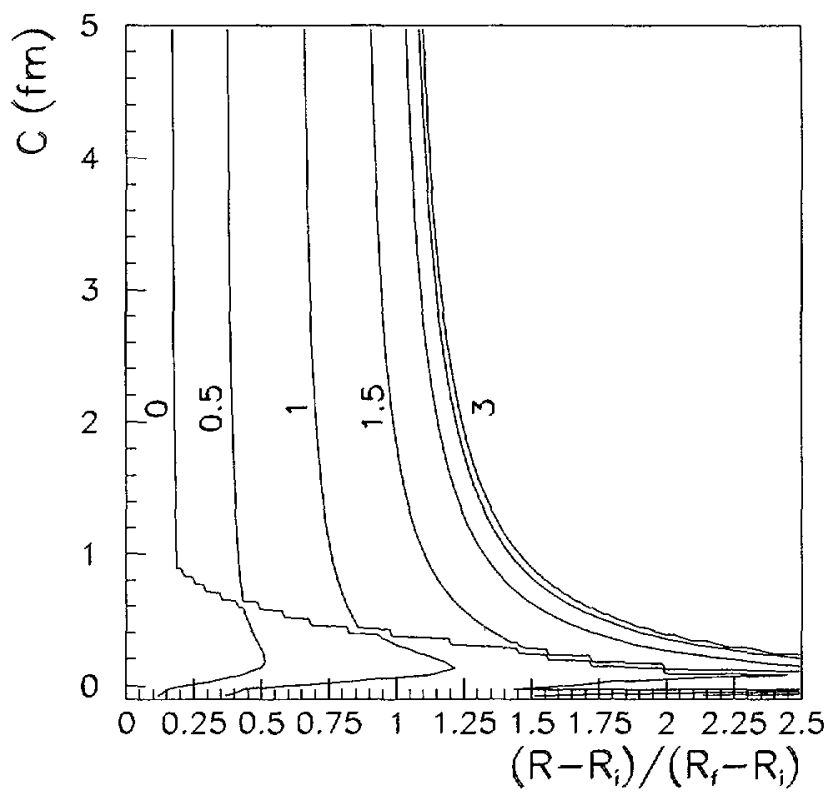

Fig. 4. - Courbes de niveaux pour la composante inertielle $\log \left(B_{R_{2} R_{2}} / \mu\right)$ dans le cas de la désintégration alpha du noyau parent ${ }^{234} \mathrm{U}$ par rapport aux deux coordonnées généralisées $C$ et $\left(R-R_{2}\right) /\left(R_{\mathrm{f}}-R_{2}\right)$. Les valeurs de cette composante sont nulles pour le noyau parent considéré sphérique et tendent vers l'infini quand les deux fragments obtenus après la désintégration sont bien séparés. Le pas entre deux valeurs consécutives des niveaux est de 0,5 unités. Quelques valeurs sont représentées près de certains niveaux pour guider l'œil.

[Contour plots of the inertia component $\log \left(B_{R_{2} R_{2}} / \mu\right)$ for the alpha-decay of the parent nucleus ${ }^{234} \mathrm{U}$ with respect two generalized coordinates $C$ and $\left(R-R_{2}\right) /\left(R_{\mathrm{f}}-R_{2}\right)$. The values of this component are zero for the spherical parent nucleus and tend to infinity when the two fragments are separated after the decay. The step between two levels is 0.5 units. Some values are plotted in the vicinity of some levels to guide the eyes.]

$$
\rho_{\imath \mathrm{e}}=3 e Z_{\imath} /\left(4 \pi r_{0}^{3} A_{\imath}\right)
$$

Dans ce travail, les paramètres seront : $a_{\mathrm{s}}=21,13 \mathrm{MeV}, \kappa_{\mathrm{s}}=2,3, a=0,68 \mathrm{fm}, a_{\mathrm{V}}=$ $15,9937 \mathrm{MeV}, \kappa_{\mathrm{V}}=1,927$.

Les quantités $B_{\mathrm{n} \imath}$ et $B_{\mathrm{c} \imath}$ dans les équations présentées sont dépendantes des formes nucléaires. Pour avoir un modèle macroscopique réaliste, on doit calculer les quantités d'intérêt physique avec des distributions de densité pour lesquelles la surface nucléaire est diffuse. Dans les noyaux, la distance sur laquelle la densité varie de $10 \%$ à $90 \%$ est d'environ $2,4 \mathrm{fm}$. Cette distance est comparable au rayon des noyaux très légers et est approximativement égale à $30 \%$ de la valeur des rayons des noyaux très lourds. Les corrections dues à la diffusivité est substantielle, spécialement pour les noyaux légers qui sont pris en compte par l'émission exotique. C'est la raison pour laquelle on introduit une correction pour le potentiel coulombien, due à la surface diffuse du noyau [11] :

$$
E_{\mathrm{d}}=-\frac{\rho_{\mathrm{e}}^{2}}{2} \int_{V} \int_{V} \mathrm{~d}^{3} r_{1} \mathrm{~d}^{3} r_{2}\left(1+\frac{1}{2} \frac{r_{12}}{a}\right) \frac{\mathrm{e}^{-\frac{r_{12}}{a}}}{r_{12}}
$$




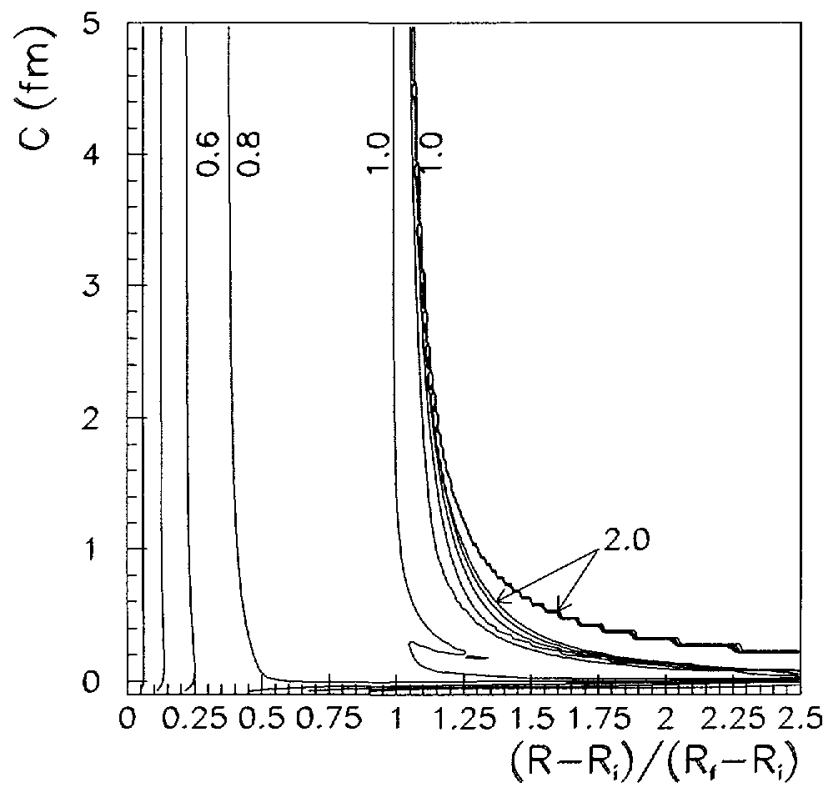

Fig. 5. - Représentation du même paramètre que sur la figure 2 pour l'émission du ${ }^{28} \mathrm{Mg}$ par le noyau parent ${ }^{234} \mathrm{U}$. Le pas entre deux valeurs consécutives des niveaux est de 0,2 unités.

[Plot of the same parameter showed in Figure 2 for the ${ }^{28} \mathrm{Mg}$ emission from the parent nucleus ${ }^{234} \mathrm{U}$. The step between two levels is 0.2 units.]

qui sera développé avec le même formalisme utilisé pour les autres termes de l'énergie (le formalisme des systèmes binaires). Le modèle ne tient pas compte de la variation de la valeur $a$ avec le nombre de masse. Pour une surface sphérique, on obtient :

$$
E_{\mathrm{d}}^{0}=-\frac{3 Z^{2} e^{2} a^{2}}{R_{0}^{3}}\left[1-\frac{15}{8} \frac{a}{R_{0}}+\frac{21}{8}\left(\frac{a}{R_{0}}\right)^{3}-\frac{3}{4} \mathrm{e}^{-\frac{2 R_{0}}{a}}\left[1+\frac{9}{2} \frac{a}{R_{0}}+7\left(\frac{a}{R_{0}}\right)^{2}+\frac{7}{2}\left(\frac{a}{R_{0}}\right)^{3}\right]\right]
$$

Pour les formes axial-symétriques et une densité de charge constante, on obtient la relation suivante qui peut être intégrée au moyen des quadratures Gauss-Legendre :

$$
\frac{E_{\mathrm{d}}}{E_{\mathrm{d}}^{0}}=b_{\mathrm{d}} \int_{-1}^{1} \int_{-1}^{1} \int_{0}^{1} F_{1}\left(\varphi, \rho_{1}, \rho_{2}, u, v\right) F_{2}\left(\varphi, \rho_{1}, \rho_{2}, u, v\right) Q\left(\varphi, \rho_{1}, \rho_{2}, u, v\right) \mathrm{d} u \mathrm{~d} v \mathrm{~d} w
$$

avec

$$
b_{\mathrm{d}}=-4 \pi^{2} \rho_{0 \mathrm{e}}^{2} a^{2} r_{0}^{3} A_{0} l^{3}
$$

où $u$ et $v$ représentent les valeurs de la coordonnée $z$ en unités $R_{0} ; l$ est la longueur, sur l'axe $z$, du noyau divisée par $2 R_{0} ; \rho_{1}=\rho\left(u R_{0}\right) / R_{0}$ et $\rho_{2}=\rho\left(v R_{0}\right) / R_{0}$ sont les variables $\rho$ en unités $R_{0}$

$$
\begin{aligned}
& F_{1}=\rho_{1}^{2}-\rho_{1} \rho_{2} \cos (\varphi)-\frac{u-v}{2} \frac{\mathrm{d} \rho_{1}^{2}}{\mathrm{~d} u} \\
& F_{2}=\rho_{2}^{2}-\rho_{1} \rho_{2} \cos (\varphi)+\frac{u-v}{2} \frac{\mathrm{d} \rho_{2}^{2}}{\mathrm{~d} v}
\end{aligned}
$$




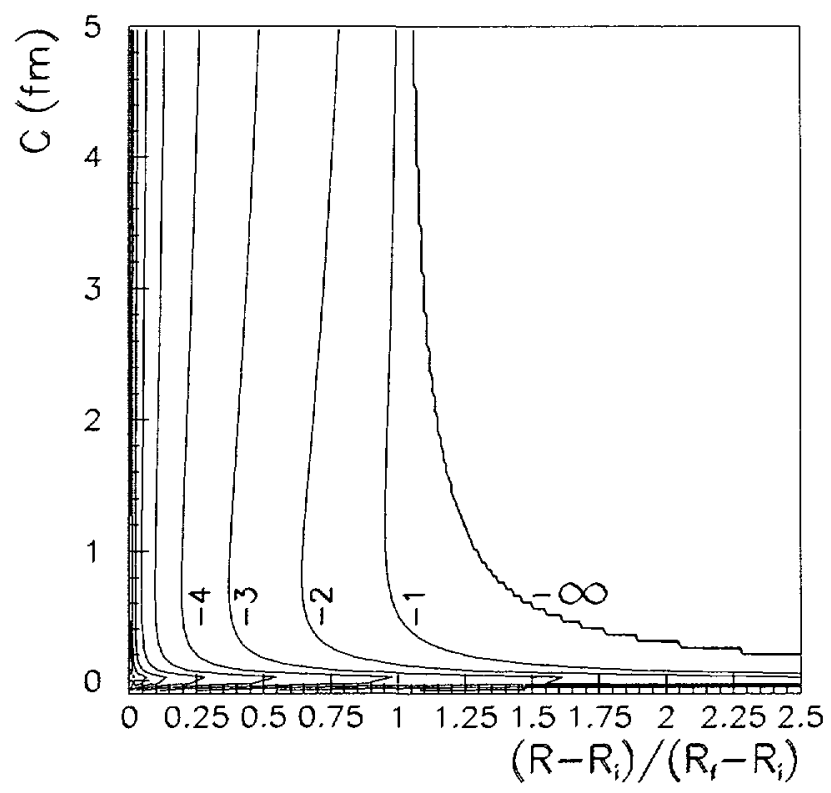

Fig. 6. - Représentation du même paramètre que sur la figure 3 pour l'émission du ${ }^{28} \mathrm{Mg}$ du noyau parent ${ }^{234} \mathrm{U}$. Le pas entre deux valeurs consécutives des niveaux est de 1 unité.

[Plot of the same parameter showed in Figure 3 for the ${ }^{28} \mathrm{Mg}$ emission from the parent nucleus ${ }^{234} \mathrm{U}$. The step between two levels is 1 unit.]

$$
\begin{aligned}
Q & =\frac{\left(\frac{R_{0} l P}{4 a}+\frac{3 P^{1 / 2}}{2}+\frac{5 a}{2 R_{0} l}\right) \exp \left(-\frac{R_{0} l P^{1 / 2}}{a}\right)-\frac{5 a}{2 R_{0} l}+P^{1 / 2}}{P^{2}} \\
P & =\rho_{1}^{2}+\rho_{2}^{2}-2 \rho_{1} \rho_{2}+(u-v)^{2} \\
w & =2 \pi \varphi .
\end{aligned}
$$

Pour des densités de charge différentes, on obtient

$$
E_{\mathrm{d}} / E_{\mathrm{d}}^{0}=\left(\rho_{1 \mathrm{e}} / \rho_{0 \mathrm{e}}\right)^{2} B_{\mathrm{d}_{1}}+\left(\rho_{1 \mathrm{e}} \rho_{2 \mathrm{e}} / \rho_{0 \mathrm{e}}^{2}\right) B_{\mathrm{d}_{12}}+\left(\rho_{2 \mathrm{e}} / \rho_{0 \mathrm{e}}\right)^{2} B_{\mathrm{d}_{2}}
$$

et

$$
\begin{aligned}
B_{\mathrm{d}_{1}} & =b_{\mathrm{d}} \int_{-1}^{z_{\mathrm{s}}} \mathrm{d} u \int_{-1}^{z_{\mathrm{s}}} \mathrm{d} v \int_{0}^{1} \mathrm{~d} w F_{1} F_{2} Q \\
B_{\mathrm{d}_{2}} & =b_{\mathrm{d}} \int_{z_{\mathrm{s}}}^{-1} \mathrm{~d} u \int_{z_{\mathrm{s}}}^{-1} \mathrm{~d} v \int_{0}^{1} \mathrm{~d} w F_{1} F_{2} Q \\
B_{\mathrm{d}_{12}} & =2 b_{\mathrm{d}} \int_{-1}^{z_{s}} \mathrm{~d} u \int_{z_{\mathrm{s}}}^{-1} \mathrm{~d} v \int_{0}^{1} \mathrm{~d} w F_{1} F_{2} Q
\end{aligned}
$$

où $z_{\mathrm{s}}$ caractérise le plan de séparation entre les deux fragments naissants en unités $R_{0}$ sur l'axe $z$.

Pour l'interaction de deux sphères disjointes, on peut utiliser la formule analytique :

$$
E_{\mathrm{d}_{12}}=-9 \frac{Z_{1} Z_{2} \mathrm{e}^{2} a_{\mathrm{d}}^{5}}{\left(R_{1} R_{2}\right)^{3}}\left[g_{1} g_{2}\left(8+\frac{R}{a_{\mathrm{d}}}-g_{2} f_{1}-g_{1} f_{2}\right] \exp \left(-\frac{R}{a_{\mathrm{d}}}\right) \frac{R}{a_{\mathrm{d}}}\right.
$$




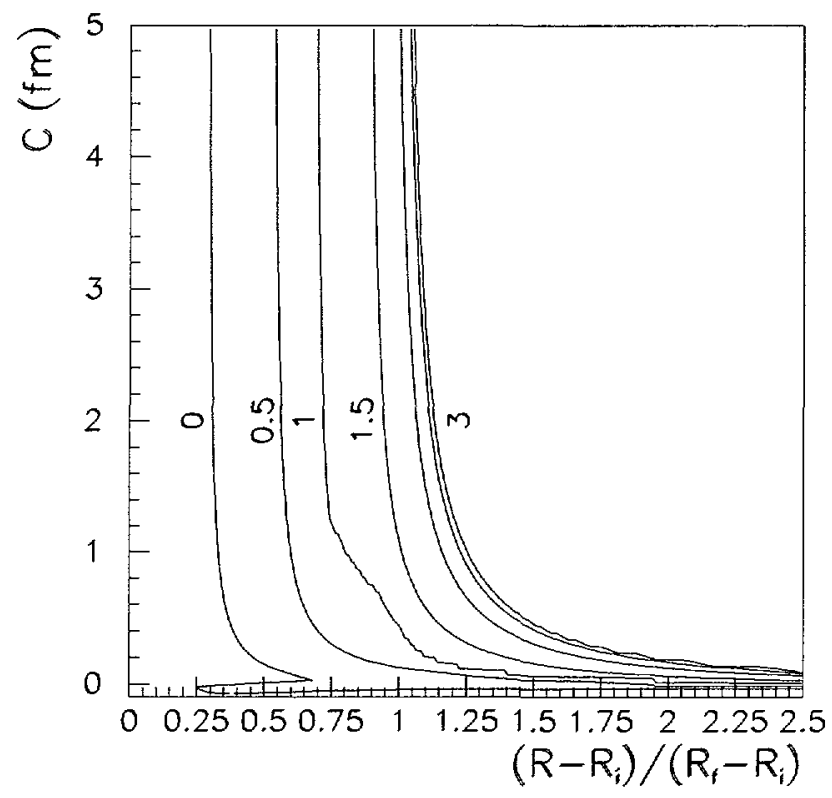

Fig. 7. - Représentation du même paramètre que sur la figure 4 pour l'émission du ${ }^{28} \mathrm{Mg}$ du noyau parent ${ }^{234} \mathrm{U}$. Le pas entre deux valeurs consécutives des niveaux est de 0,5 unités.

[Plot of the same parameter showed in Figure 4 for the ${ }^{28} \mathrm{Mg}$ emission from the parent nucleus ${ }^{234} \mathrm{U}$. The step between two levels is 0.5 units.]

avec

$$
\begin{aligned}
& g_{\imath}=\frac{R_{2}}{a_{\mathrm{d}}} \operatorname{ch}\left(\frac{R_{2}}{a_{\mathrm{d}}}\right)-\operatorname{sh}\left(\frac{R_{2}}{a_{\mathrm{d}}}\right), \quad i=1,2 \\
& f_{\imath}=\left(\frac{R_{\imath}}{a_{\mathrm{d}}}\right)^{2} \operatorname{sh}\left(\frac{R_{2}}{a_{\mathrm{d}}}\right)
\end{aligned}
$$

On doit aussi ajouter la contribution de l'énergie de volume

$$
E_{\mathrm{V}}=E_{\mathrm{V}_{1}}+E_{\mathrm{V}_{2}}-E_{\mathrm{V}_{0}}
$$

où

$$
E_{\mathrm{V}_{2}}=-a_{\mathrm{V}}\left[1-\kappa_{\mathrm{V}}\left(N_{2}-Z_{\imath}\right)^{2} / A_{2}^{2}\right]
$$

L'énergie d'interaction pour deux noyaux sphériques peut être obtenue à l'aide de relations analytiques. L'énergie de déformation totale devient :

$$
E\left(q_{1}, q_{2}, q_{3}\right)=E_{\mathrm{n}}+E_{\mathrm{c}}+E_{\mathrm{d}}+E_{\mathrm{V}}-E_{0}
$$

L'importance du terme qui simule la diffusivité sur la surface pour l'énergie Coulombienne peut être appréciée en calculant la chaleur de désintégration pour les trois types de processus nucléaires traités dans cet article. Les valeurs expérimentales de la chaleur de désintégration $Q_{\text {exp }}$ sont, respectivement, 4,86 MeV, $74,13 \mathrm{MeV}$ et $197,17 \mathrm{MeV}$ [12] pour la désintégration $\alpha$, 


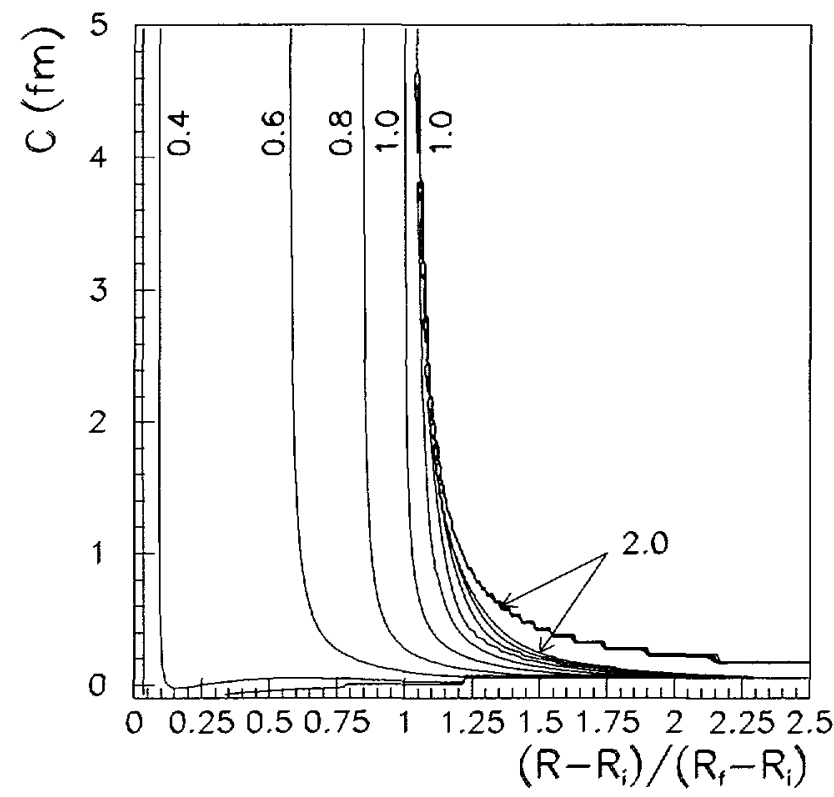

Fig. 8. - Représentation du même paramètre que sur la figure 2 pour la fission du noyau parent ${ }^{234} \mathrm{U}$ avec, pour fragment léger, ${ }^{100} \mathrm{Zr}$. Le pas entre deux valeurs consécutives des niveaux est de 0,2 unités. [Plot of the same parameter showed in Figure 2 for the cold fission of the parent nucleus ${ }^{234} \mathrm{U}$ with light fragment ${ }^{100} \mathrm{Zr}$. The step between two levels is 0.2 units.]

l'émission $\mathrm{du}{ }^{28} \mathrm{Mg}$ et la fission froide - avec, pour fragment léger, ${ }^{100} \mathrm{Zr}$ - du noyau parent ${ }^{234} \mathrm{U}$. Les valeurs $Q_{\mathrm{th}}^{*}$, calculées sans le terme qui simule la diffusivité sur la surface pour l'énergie coulombienne, sont, pour les mêmes processus : 7,25 MeV, 76,04 MeV et 206,49 MeV, tandis qu'on obtient, pour $Q_{\text {th }}$ (avec le terme de diffusivité), $6,70 \mathrm{MeV}, 74,57 \mathrm{MeV}$ et $204,29 \mathrm{MeV}$, respectivement. Ces dernières valeurs sont plus proches des données expérimentales, donc le modèle devient plus réaliste.

Enfin, on ajoute une correction phénoménologique

$$
E_{\text {corr }}=\left(Q_{\text {th }}-Q_{\text {exp }}\right) V_{2}(R) / V_{2 \mathrm{f}}
$$

destinée à reproduire exactement la valeur expérimentale de la chaleur de fission $Q_{\text {exp }}$. $Q_{\text {th }}$ est la valeur théorique obtenue dans le cadre de ce modèle. Cette correction est considérée proportionnelle au volume du fragment émis.

Les lignes équipotentielles pour l'énergie de déformation obtenues avec ce modèle sont représentées dans les figures 11,12 et 13 pour la désintégration alpha, l'émission du ${ }^{28} \mathrm{Mg}$ et la fission froide - avec, pour fragment léger, ${ }^{100} \mathrm{Zr}$ - du parent ${ }^{234} \mathrm{U}$ par rapport à l'élongation normalisée et le col. On peut voir que l'énergie de déformation décroît dans la région des formes gonflées, donc, pour les faibles valeurs du paramètre macroscopique $C$. Il est évident que, si on néglige les paramètres de masse, du point de vue purement énergétique, la trajectoire de fission dans l'espace des configurations doit être simulée par des formes nucléaires gonflées. 


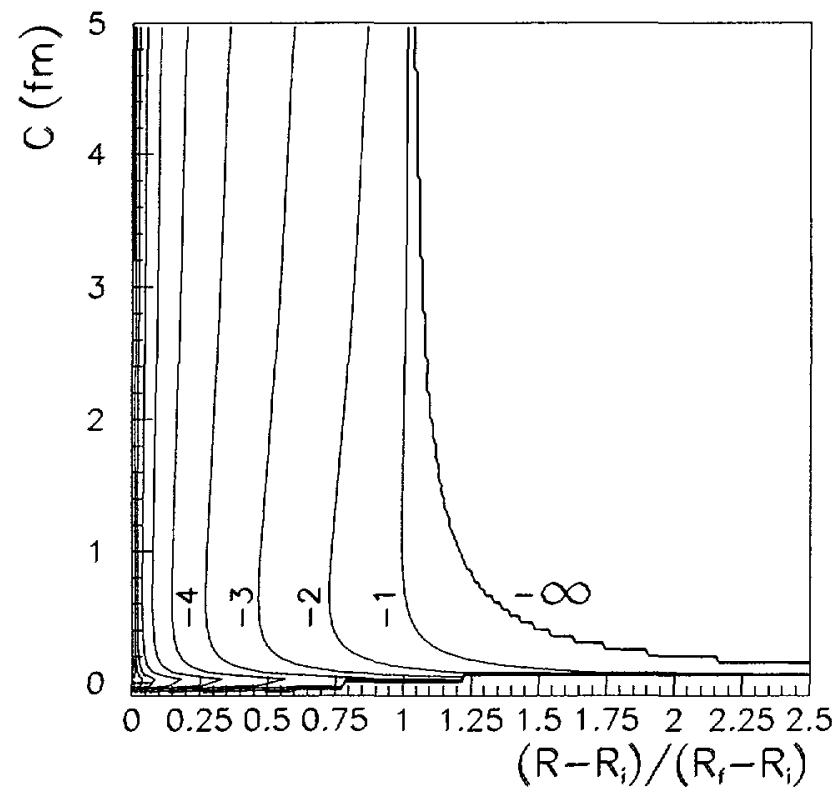

Fig. 9. - Représentation du même paramètre que sur la figure 3 pour la fission du noyau parent ${ }^{234} \mathrm{U}$ avec, poux fragment léger, ${ }^{100} \mathrm{Zr}$. Le pas entre deux valeurs consécutives des niveaux est de 1 unité.

[Plot of the same parameter showed in Figure 3 for the cold fission of the parent nucleus ${ }^{234} \mathrm{U}$ with light fragment ${ }^{100} \mathrm{Zr}$. The step between two levels is 1 unit.]

\section{Minimisation de l'intégrale d'action}

La pénétrabilité $P$ de la barrière de potentiel pour la fission dans l'espace multidimensionnel des configurations est calculée avec la méthode semi-classique WKB [13]. La région d'intérêt dans laquelle on obtient la pénétrabilité est classiquement interdite. L'intégrale d'action est calculée le long d'une trajectoire classique pour une énergie donnée qui relie un point qui caractérise l'état fondamental du noyau parent et le point de sortie de la barrière multidimensionnelle.

Si on suppose un système de deux sphères en contact pour la forme qui caractérise la scission (approximation naturelle tenant compte du fait que, pour les désintégrations étudiées, les énergies d'excitation des fragments sont négligeables), la pénétrabilité le long de la trajectoire peut être exprimée comme le produit de deux pénétrabilités :

$$
P=P_{\mathrm{ov}} P_{\mathrm{s}}=\exp \left[-\left(K_{\mathrm{ov}}+K_{\mathrm{s}}\right)\right] .
$$

Ici $K_{\mathrm{s}}$ représente l'intégrale d'action pour les deux fragments séparés tandis que $K_{\mathrm{ov}}$ est relative au système dans l'état de préscission.

$$
K_{\mathrm{ov}}=\frac{2}{\hbar} \int_{R_{\imath}}^{R_{\mathrm{s}}} \sqrt{2 B\left(R, C, R_{2}, \frac{\partial C}{\partial R}, \frac{\partial R_{2}}{\partial R}\right) E\left(R, C, R_{2}\right)} \mathrm{d} R=\frac{2}{\hbar} \int_{R_{\mathrm{s}}}^{R_{\mathrm{s}}} F \mathrm{~d} R
$$

où $R_{2}=r_{0}\left(A_{0}^{1 / 3}-A_{2}^{1 / 3}\right)$ est un point de rebroussement, $R_{\mathrm{s}}=r_{0}\left(A_{1}^{1 / 3}+A_{2}^{1 / 3}\right)$ est le point de scission, $A_{0}, A_{1}, A_{2}$ étant les nombres de masse du noyau parent, du noyau descendant et du fragment émis. 


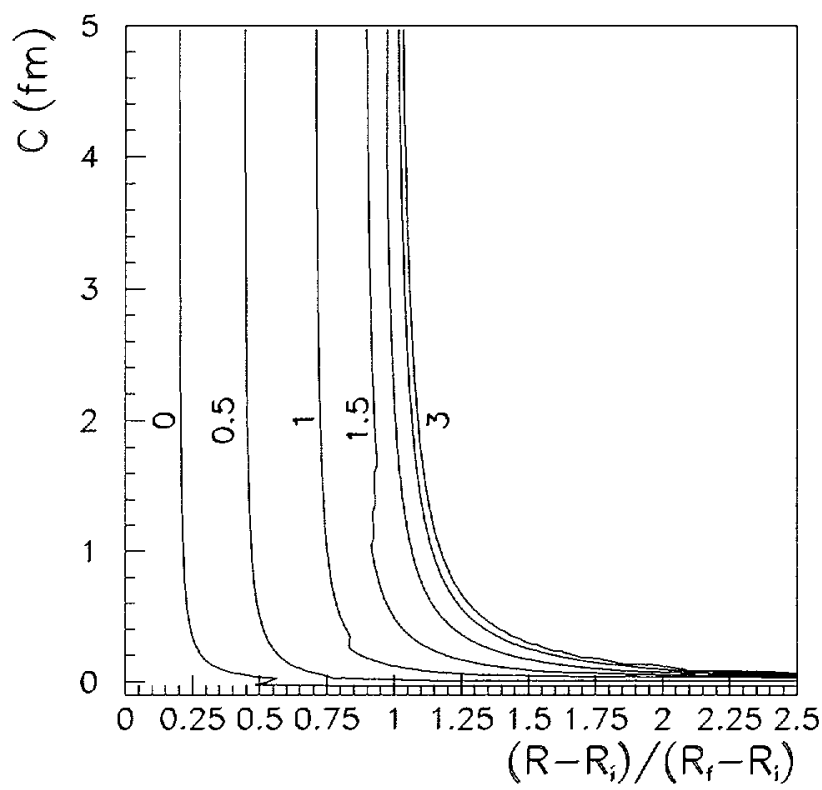

Fig. 10. - Représentation du même paramètre que sur la figure 4 pour la fission du noyau parent ${ }^{234} \mathrm{U}$ avec, pour fragment léger, ${ }^{100} \mathrm{Zr}$. Le pas entre deux valeurs consécutives des niveaux est de 0,5 unités.

[Plot of the same parameter showed in Figure 4 for the cold fission of the parent nucleus ${ }^{234} \mathrm{U}$ with light fragment ${ }^{100} \mathrm{Zr}$. The step between two levels is 0.5 units.]

Le but des calculs régis par le principe de moindre action est d'obtenir une trajectoire parmi le nombre infini de trajectoires possibles, qui correspond à la plus petite valeur de l'intégrale d'action calculée dans le domaine, classiquement interdit, qui réunit les deux points de rebroussement $R_{\mathrm{s}}$ et $R_{\imath}$ et, pour ce cas particulier, pour tous les points qui peuvent caractériser l'état fondamental.

Heureusement, le point de scission dans l'espace des configurations étant connu (configuration de deux sphères tangentes), le problème s'en trouve beaucoup simplifié car, on n'a plus besoin d'essayer de minimiser par rapport à tous les points de rebroussement qui peuvent caractériser le point de sortie de la barrière de potentiel multidimensionnelle. Donc, pour $R=R_{\mathrm{f}}$, on sait que $R_{3}=0$ fm et $R_{2}=R_{2 \mathrm{f}}$.

A vec notre paramétrisation, dans la région de superposition des fragments, l'inertie le long de la trajectoire est donnée par la formule:

$$
\begin{aligned}
B\left(R, C, R_{2}, \partial C / \partial R, \partial R_{2} / \partial R\right)= & B_{R R}+2 B_{R C} \frac{\partial C}{\partial R}+B_{C C}\left(\frac{\partial C}{\partial R}\right)^{2} \\
& +2 B_{R, R_{2}} \frac{\partial R_{2}}{\partial R}+B_{R_{2} R_{2}}\left(\frac{\partial R_{2}}{\partial R}\right)^{2}
\end{aligned}
$$

Quand $C>1 \mathrm{fm}^{-1}$, il est préférable d'utiliser $R_{3}$ comme paramètre caractérisant le col. Maintenant, pour minimiser l'intégrale d'action, on transforme la fonctionnelle $K_{\text {ov }}$ en une fonction. Cela est possible en remplaçant l'intégrale par une somme donnée par des quadratures [7]. Dans nos calculs, deux quadratures Gauss-Legendre (une avec 20 points et l'autre avec 32 


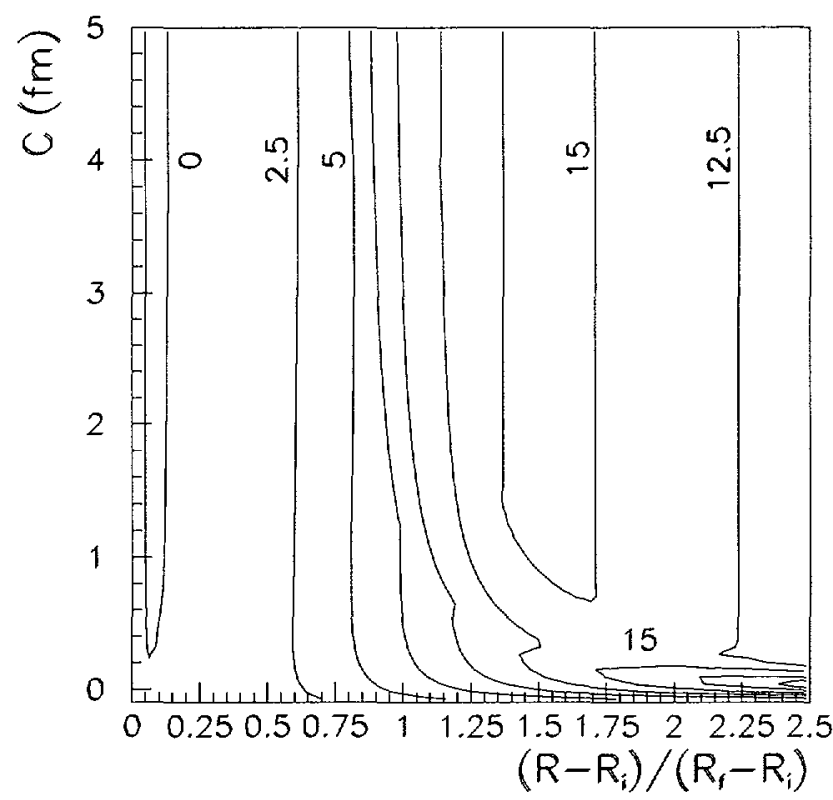

Fig. 11. - Énergie de déformation en $\mathrm{MeV}$ calculée pour la désintégration $\alpha$ du noyau ${ }^{234} \mathrm{U}$ dans le cadre du modèle des systèmes binaires avec des densités de charge différentes par rapport aux deux paramètres macroscopiques qui caractérisent l'élongation et le col. Le pas entre deux valeurs consécutives des niveaux est de 2,5 MeV. Quelques valeurs de l'énergie de déformation sont données près des lignes équipotentielles pour guider l'œil.

[Deformation energy in $\mathrm{MeV}$ calculated for the $\alpha$ decay of the ${ }^{234} \mathrm{U}$ nucleus in the framework of the binary system with different charge densities model with respect the two macroscopic parameters which describe the elongation and the necking. The step between two levels is $2.5 \mathrm{MeV}$. Some values are given to guide the eyes.]

points) ont été utilisées simultanément. Cette procédure a permis de définir la trajectoire $C=C\left(R\right.$ ) (ou $R_{3}=R_{3}(R)$ ) avec un jeu de 52 variables indépendantes $C_{\imath \jmath}$ (ou $R_{3 \imath}$ ) fixées dans les points de maillage ou nouds $R_{\imath \jmath}$. Les deux quadratures sont caractérisées par les indices $(i=1 ; j=1,2, . ., 32)$ et $(i=2 ; j=1,2, \ldots, 20)$. La trajectoire est alors obtenue par interpolation par splines parmi ces points réarrangés par ordre croissant, procédure à laquelle on ajoute la condition relative à la paramétrisation de la forme nucléaire donnée par deux sphères tangentes à la scission, c'est-à-dire, le point $R_{3}=0 \mathrm{fm}$ pour $R=R_{\mathrm{f}}$; ce qui permet de calculer les dérivées $\mathrm{d} C / \mathrm{d} R$ pour tous les points $R_{\imath 3}$, valeurs nécessaires au calcul des inerties. La minimisation est ensuite effectuée numériquement en prenant un jeu de 52 valeurs initiales pour $C_{\imath \jmath}$ ou $R_{3 \imath \jmath}$. Pour chaque jeu de valeurs initiales, on trouve un minimum local ; mais on retient, en fin de compte, le minimum qui donne la valeur optimale de l'intégrale.

\section{Résultats}

Il est connu que la région des faibles déformations ne présente pas un grand intérêt pour ce genre de calculs car les faibles valeurs de l'énergie de déformation sont multipliées par des quantités négligeables que représentent les masses effectives. Les calculs numériques montrent que plus de $75 \%$ de la valeur totale de l'intégrale d'action $K_{\text {ov }}$ provient de la région $R_{n} \subset[0,5-1] \mathrm{du}$ domaine d'élongation se trouvant au voisinage du point de scission. 


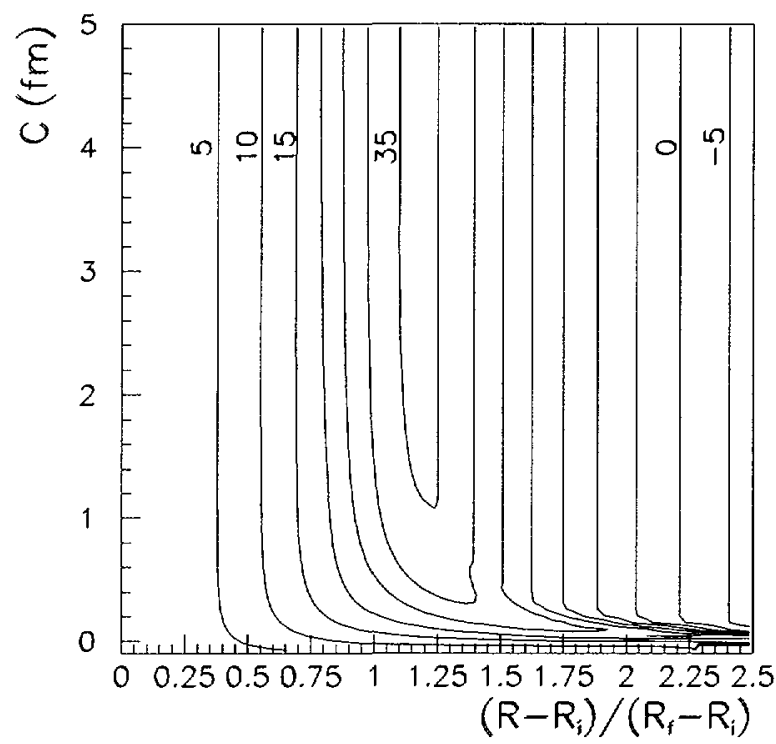

Fig. 12. - Représentation du même paramètre que sur la figure 11 pour l'émission de ${ }^{28} \mathrm{Mg}$ du noyau ${ }^{234} \mathrm{U}$. Le pas entre deux valeurs consécutives des niveaux est de $5 \mathrm{MeV}$.

[Plot of the same parameter showed in Figure 11 for the ${ }^{28} \mathrm{Mg}$ emission from the ${ }^{234} \mathrm{U}$ nucleus. The step between two levels is $5 \mathrm{MeV}$.]

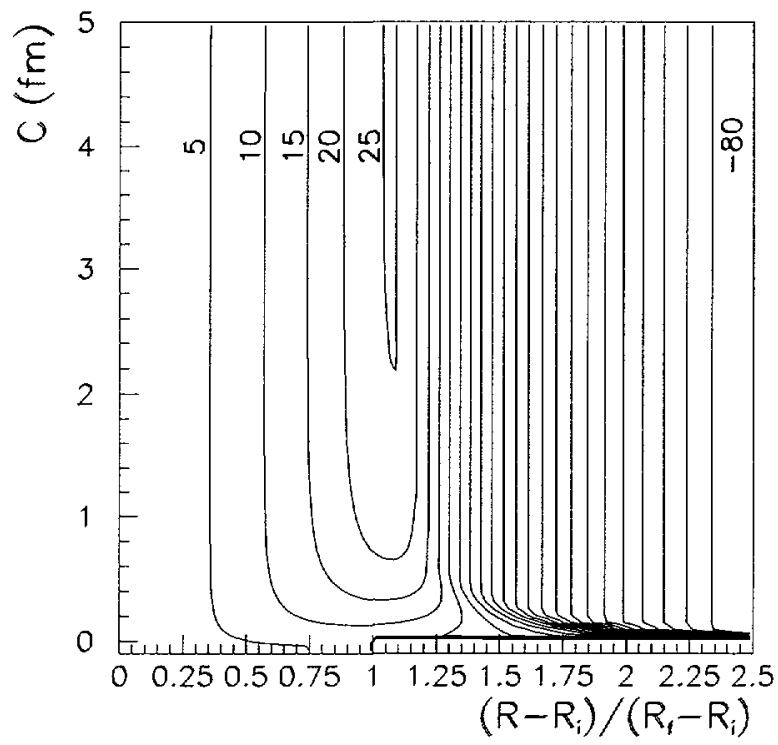

Fig. 13. - Représentation du même paramètre que sur la figure 11 pour la fission froide - avec, pour fragment léger, ${ }^{100} \mathrm{Zr}-\mathrm{du}$ noyau ${ }^{234} \mathrm{U}$. Le pas entre deux valeurs consécutives des niveaux est de $5 \mathrm{MeV}$.

[Plot of the same parameter showed in Figure 11 for the cold fission of the ${ }^{234} \mathrm{U}$ nucleus with light fragment ${ }^{100} \mathrm{Zr}$. The step between two levels is $5 \mathrm{MeV}$.] 


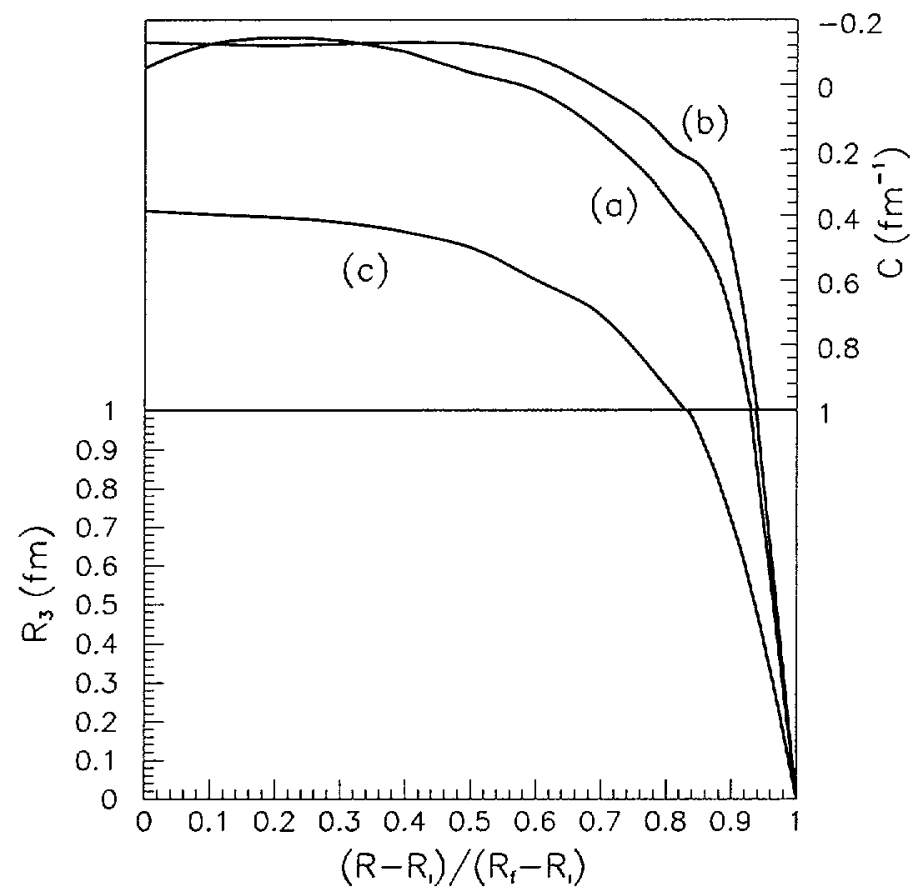

Fig. 14. - Trajectoires de moindre action pour (a) la fission froide avec, pour fragment léger, ${ }^{100} \mathrm{Zr}$, (b) l'émission spontanée du ${ }^{28} \mathrm{Mg}$ et (c) la désintégration alpha du noyau ${ }^{234} U$ par rapport à l'élongation normalisée. La trajectoire est décrite avec les valeurs du paramètre $R_{3}$ quand le rayon du col a des valeurs plus petites que $1 \mathrm{fm}$, et avec le paramètre $C$ pour les autres valeurs qui caractérisent le col.

[Least action trajectories for (a) cold fission with light fragment ${ }^{100} \mathrm{Zr}$, (b) ${ }^{28} \mathrm{Mg}$ emission and (c) alpha-decay of the ${ }^{234} \mathrm{U}$ nucleus with respect the normalized elongation. The trajectory is described by the values of $R_{3}$ when the neck radius is smaller than $1 \mathrm{fm}$, and with $C$ otherwise.]

Des calculs pour la désintégration alpha, l'émission spontanée du ${ }^{28} \mathrm{Mg}$ et la fission froide - avec, pour fragment léger, ${ }^{100} \mathrm{Zr}$ - du noyau parent ${ }^{234} \mathrm{U}$ ont été effectués. C'est le premier noyau pour lequel on a détecté expérimentalement ces trois modes de désintégration [14]. La figure 14 reproduit les trajectoires de fission. La trajectoire optimale pour la désintégration alpha présente un rayon $R_{3}$ très petit qui caractérise le col. Par conséquent, pour les très grandes asymétries de masse, une paramétrisation de la forme nucléaire donnée par un système de deux sphères qui se recouvrent doit approximer très bien ce type de processus. Une situation différente apparaît pour l'émission exotique et la fission froide où, dans la première étape du processus, les formes en diamant sont favorisées. Pour la fission froide, le rayon du col commence à diminuer dans une proximité du point de scission plus prononcée que pour l'émission exotique. Ces résultats sont en bon accord avec les comportements déjà publiés. Les formes nucléaires qui correspondent aux trajectoires optimales sont reproduites sur la figure 15.

Pour les temps de vie du noyau parent ${ }^{234} \mathrm{U}$, on a, d'après les références $[5,14], T_{1 / 2}=$ $2,45 \times 10^{5}$ années pour la désintégration alpha, $T_{1 / 2}=1,1 \times 10^{18}$ années pour l'émission $\mathrm{du}{ }^{28} \mathrm{Mg}$ et $T_{1 / 2}=1,9 \times 10^{16}$ années pour la fission froide. Le modèle de fission conduit à 


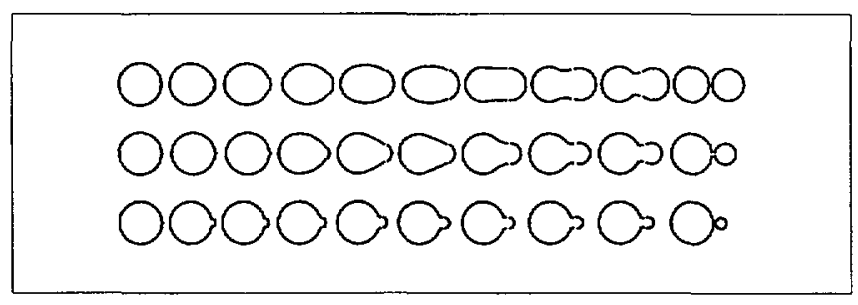

Fig. 15. - Formes nucléaires déterminées le long de la trajectoire de moindre action pour la fission froide avec, pour fragment léger, ${ }^{100} \mathrm{Zr}$ (en haut), l'émission de ${ }^{28} \mathrm{Mg}$ (au milieu) et la désintégration alpha (bas) du même noyau parent, ${ }^{234} \mathrm{U}$.

[Nuclear shapes along optimum fission paths for cold fission with ${ }^{100} \mathrm{Zr}$ light fragment, ${ }^{28} \mathrm{Mg}$ radioactivity and alpha-decay of $\left.{ }^{234} \mathrm{U}\right]$.

l'estimation du temps de vie selon la formule:

$$
T_{1 / 2}=\frac{h \ln 2}{2 E_{\mathrm{v}}} \exp (K)
$$

où $E_{\mathrm{v}}$ représente l'énergie de vibration du point zéro et $\exp (K)$ nous donne la pénétrabilité totale à travers la barrière multidimensionnelle.

Conformément à ce modèle, si on ne fait aucune hypothèse sur la variation de la valeur de $E_{\mathrm{v}}$ par rapport au nombre de masse du noyau léger, le rapport des temps de vie relatifs à la désintégration alpha et à la radioactivité par émission du noyau ${ }^{28} \mathrm{Mg}$ sera égal à :

$$
b_{\alpha / M_{g}}=\frac{\exp \left(K_{\alpha}\right)}{\exp \left(K_{M_{g}}\right)}
$$

Donc, pour juger la validité du modèle par rapport à l'expérience, on doit calculer les valeurs de $K$. Les valeurs théoriques de l'intégrale d'action ont été obtenues par intégration numérique après avoir interpolé les trajectoires au moyen de fonctions splines. Pour comparer, on a déduit aussi les valeurs de l'intégrale d'action pour le cas particulier de deux sphères qui se recouvrent pour les trois processus nucléaires étudiés. Dans ce cas, on a obtenu les résultats théoriques suivants : $K_{\text {ov }}=93,89 ; 41,63 ; 6,0$, respectivement, pour la fission - avec, pour fragment léger, ${ }^{100} \mathrm{Zr}$ - , l'émission du ${ }^{28} \mathrm{Mg}$ et la radioactivité alpha. Pour la pénétrabilité de la barrière extérieure (après la scission), on a obtenu, respectivement, : $K_{\mathrm{s}}=56,102 ; 76,51$ et 78,06 . Pour les trajectoires représentées sur la figure 14 , on a obtenu les valeurs suivantes pour $K_{\text {ov }}^{\text {optimal }}=$ 64,$43 ; 34,56$ et 5,11 , respectivement, pour la fission - avec, pour fragment léger, ${ }^{100} \mathrm{Zr}-$ l'émission $d u{ }^{28} \mathrm{Mg}$ et la désintégration alpha. Avec ces dernières valeurs de la pénétrabilité, on peut calculer le rapport des temps de vie sans prendre en compte aucune dépendance semiempirique pour l'énergie de vibration du point zéro. On obtient la valeur théorique $\log \left(b_{\alpha / \mathrm{Mg}}^{\text {theo }}\right)=$ $-12,11$. La valeur expérimentale est $\log \left(b_{\alpha / \mathrm{Mg}}^{\exp }\right)=-12,75$. Ces deux valeurs présentent un très bon accord ce qui prouve la validité du modèle et nous conduit à faire une prédiction pour le temps de vie pour la fission froide - avec, pour fragment léger, ${ }^{100} \mathrm{Zr}$ - considérant que le rapport théorique obtenu $\log \left(b_{\alpha / Z_{\mathrm{r}}}^{\text {theo }}\right)=-16,22$ est correct. On obtient $\log \left(T_{1 / 2}[\mathrm{~s}]\right)=26,22$ (ici, le temps de vie est estimé en secondes) pour la fission froide - avec, pour fragment léger, ${ }^{100} \mathrm{Zr}$ - du noyau parent ${ }^{234} \mathrm{U}$ tandis que la valeur expérimentale est considérée plus grande que 23,68 . 


\section{Remerciements}

Nous remercions chaleureusement R. Bimbot et $F$. Clapier pour le vif intérêt témoigné à ce travail, ainsi que pour l'aide constante qu'ils nous ont apportée et sans laquelle il n'aurait pu être mené à bien.

\section{Bibliographie}

[1] Poenaru D.N. and Ivascu M., Fission at very large mass and charge, Critical Phenomena in Heavy Ion Physics (A. Raduta et G. Stratan, Eds., ICEFIZ, Bucarest, 1980) p.743.

[2] Sandulescu A., Poenaru D.N. and Greiner W., New type of decay of heavy nuclei intermediate between fission and alpha-decay, Sov. J. Part. Nucl. 11 (1980) 528.

[3] Depta K., Maruhn J.A., Wang Hou-Ji, Sandulescu. A. and Greiner W., On the necking-in process in cluster decays, Int. J. Mod. Phys. A 5 (1990) 3901.

[4] Mirea M., Poenaru D.N. and Greiner W., Optimized fission paths as solutions of differential equations, Nuovo Cimento 105A (1992) 571.

[5] Mirea M., Poenaru D.N. and Greiner W., Threedimensional dymanics of nuclear decay modes, Z.Phys. A 349 (1994) 39.

[6] Benfoughal T. et Mirea M., Dynamique de la désintégration pour trois types de processus nucléaires, J.Phys. III France 5 (1995) 1115.

[7] Poenaru D.N., Mirea M., Greiner W., Cata I. and Mazilu D., Neck influence on dynamics of cluster radioactivities and cold fission, Mod. Phys. Lett. A 26 (1990) 2101.

[8] Poenaru D.N., Ivascu M., Ivascu I., Mirea M., Greiner W., Depta K. and Renner W., Werner-Wheeler dynamics of cluster emission and cold fission, Conference: 50 Years with Nuclear Fission (J.W. Behrens and A.D. Carlson, Eds., American Nuclear Society, Lagrange Park, 1989) p. 617.

[9] Krappe H.J., Nix J.R. and Sierk A.J., Unified nuclear potential for heavy-ion elastic scattering, Phys.Rev.C 20 (1979) 992.

[10] Poenaru D.N., Ivascu M. and Mazilu D., Folded Yukawa-plus-exponential model PES for nuclei with different charge densities, Comp.Phys.Comm. 19 (1980) 205.

[11] Davies K.T.R. and Nix J.R., Calculation of moments, potentials, and energies for an arbitrarily shaped diffuse-surface nuclear density distributions, Phys. Rev. C 14 (1976) 1977.

[12] Audi G. and Wapstra A.H., The 1993 atomic mass evaluation I: atomic mass table, Nucl. Phys. A 565 (1993) 1.

[13] Brack M., Damgaard J., Jensen A., Pauli H.C., Strutinsky V.M. and Wong G.Y., Funny Hills: the shell correction approach to nuclear shell effects and its applications to the fission process, Rev. Mod. Phys. 44 (1972)320.

[14] Shicheng Wang, Price P.B., Barwick W.S., Moody K.J. and Hulet E.K., Radioactive decay of ${ }^{234} \mathrm{U}$ via $\mathrm{Ne}$ and $\mathrm{Mg}$ emission, Phys. Rev. C 36 (1987) 2717. 